\title{
Fractional Discrimination for Texture Image Segmentation
}

\author{
J. You ${ }^{1} \quad$ S. Hungnahally ${ }^{2}$ A. Sattar ${ }^{1}$ \\ ${ }^{1}$ School of Computing and Information Technology \\ ${ }^{2}$ School of Microelectronic Engineering \\ Griffith University, Nathan Campus \\ Brisbane, Queensland, Australia 411
}

\begin{abstract}
Texture image segmentation plays an important role in texture analysis. This paper presents an approach to image segmentation by texture classification based on fractional discrimination functions. The idea behind this method is to enhance the texture edge points by means of image decomposition and contextual filtering in terms of the proposed fractional function. In addition, such function is described in a unified form with three-parameters. The parameters determine the global scale in conjunction with local scales for feature identification. Our experimental results show that texture features can be effectively extracted on the basis of the selective fractional discrimination function.

Keywords and phrases: fractional discrimination function, feature extraction, image segmentation, texture classification.
\end{abstract}

\section{Introduction}

An important component in image analysis is segmentation of image into meaningful regions with certain properties. Texture provides a high-order description of the local image content. Image segmentation by texture property has been studied by many researchers[1]. Textured regions may often be segmented on the basis of texture feature extraction. The central problem concerned is to find an efficient and effective approach to extract texture feature points so as to identify the boundaries of different regions invariant of scales and orientations. Conventional edge detection techniques based on detection of discontinuities in pixel properties are not suitable for distinguishing boundaries between differently textured regions because texture is characterised by its local features over some neighbourhood rather than in pixel scales. Therefore, an operator sensitive to different textures is required to enhance some texture components and to suppress others for texture edge detection. In contrast to the traditional methods, our solution involves the introduction of fractional functions as discrimination functions to perform robust (in the presence of noise), selective (band limited) and contextual feature extraction. In our previous work[4] we proposed a mask tuning scheme which extended Laws' texture energy concept[2] and Benke et al.'s work[3] for dynamic texture feature extraction. In the work reported here we further define a generalised framework to encompass the existing functions and masks for feature extraction. The use of fractional discrimination for the extraction of embedded features within random textures provides a systematic and iterative method to search for dynamic features for image analysis.

The idea behind this method is to enhance the feature points by means of image decomposition and contextual filtering on the basis of the proposed fractional function. In addition, such function is described in a unified form with three-parameters, where the function performs differentiation with inherent aggregation. Consequently, unlike the existing approaches[5] which mostly rely on different individual operators, our study simplifies the feature extraction operation in a general format based on the perceptual/scale-space properties of the visual receptive fields, which provides an effective approach to feature extraction and suitable to many vision tasks.

In this paper we describe a simple but reliable approach to detect texture feature points for segmentation. Section 2 describes the proposal of a generalised framework of discrimination functions based on the visual receptive field models. Section 3 details our extension of fractional discrimination function for robust texture feature extraction, Section 4 highlights its application to image segmentation. Finally, our conclusions are presented in Section 5 .

\section{Discrimination Functions and Edge Features}

The classical approaches to edge detection, reviewed by Davis[5], include high emphasis filtering, 
gradient operations, optimal techniques and a dynamic programming formulation of the edge detection problem. Several notable new techniques for edge detection have been developed, such as new classes of operators, Marr's edge detection theory, multidimensional edge detection, and the facet model-based approach. However, there is lack of a generalised framework of discriminations for edge detection although some initial work was reported by Koenderink[6]. We aim to apply the visual receptive field models to generate robust and selective discrimination functions in a general form which encompass the traditional individual mask operators. The key points regarding such an extension are summarized as below.

\subsection{Visual Receptive Field Models}

Biological visual receptive fields are regarded as robust processing units used in biological systems for information extraction. In the past studies in modeling visual receptive field were mainly concerned with two objectives: to explain the principles of biological visual systems; and to apply the models for information extraction in artificial vision systems. In general, the modeling is based on the assumption that the neural system is sensitive to derivatives in perceiving any physical phenomena. Thus, features in visual environments can be described in terms of differentiation. In accordance with the bipolar nature of the receptive fields, we are concerned with the generation of discrimination functions for emulation. As a result, the receptive fields are modeled as a family of filters which perform low-pass filtering, smoothing and differentiation simultaneously.

\subsection{Generalised Discrimination Function} vs. Mask Operators

Basically, the idea underlying most edge-detection techniques is the computation of local derivative operator. Many authors have proposed digital approximations to such process(Robert's operator, Sobel's operator, Prewitt's operator, Marr's LOG operator, Haralick's zero-crossing operator, Canny's edge detector). However, the design of these operators was not based on the biological visual receptive fields and these individual operators were not represented in a general form. Although some initial work has been done by Koenderink who applied the convolution of certain receptive field profiles with image to yield the partial derivatives of the retinal illumination for feature extraction, the application of his work is limited due to his assumption of linear image plane. We further introduce generalised discrimination functions to identify edge features with more flexibility.

The generalised discrimination functions $g(\xi)$ are defined within the bounds of a multi-dimensional coordinate system $\xi$. In the case of one-dimensional timedomain signals, $\xi$ represents time $t$; for time-invariant images, $\xi$ refers to the spatial coordinates $(x, y)$; and for time-dependent images, $\xi$ means $(x, y, t)$. Let $f(\xi)$ be a multi-dimensional periodic or aperiodic function defined over $\xi \in[-\infty, \infty]$ in terms of their zero crossings or zero nodes (with at least one zerocrossing). Therefore, the generalised discrimination function $g(\xi)$ is defined as

such that

$$
g(\xi)= \begin{cases}f(\xi) & \text { if } \xi \in\left[k_{m}, k_{n}\right] \\ 0 & \text { otherwise }\end{cases}
$$

$$
\int_{-\infty}^{\infty} g(\xi) d \xi=\int_{k_{m}}^{k_{n}} f(\xi) d \xi=0
$$

where $k$ represents a zero crossing $\left(k_{m}\right.$ and $k_{n}$ refers to $m^{t h}$ and $n^{\text {th }}$ zero crossing respectively), $m, n$ are any integers and $m<n$.

By introducing such a general form, discriminations for feature extraction can be generalised with any order, size and shape or a combination of the three. All such discrimination functions perform differentiation with inherent aggregation. In addition, these functions are orthogonal to the aggregation weighting functions.

\section{Fractional Discrimination Functions for Texture Feature Extraction}

In the previous section we introduced a general form of discrimination functions with which the existing different varieties of the conventional operators can be derived. In this section we further extend the concept of fractional derivatives so as to propose effective fractional discrimination functions for robust texture feature extraction.

The concept of fractional derivatives is the approximation of the non-integer derivatives to a continuous domain with the converge to the first-, second- and finite-order derivatives. The fractional derivative of a constant is not always equal to zero [8]. Based on the different approaches to define such fractional derivatives, different fractional discrimination functions will be developed. In the work reported here, we adopted the following idea in the area of fractional calculus to define the fractional differention.

Let $f(\xi)$ represent any function in the domain of $\xi$, $D^{\nu} f(\xi)$ refer to as the fractional differention of function $f(\xi)$ at the order of $\nu$, where $\nu$ is any real positive number. If gamma function is used, we have

$$
D^{-\nu} f(\xi)=\frac{1}{\Gamma(\nu)} \int_{c}^{\xi}(\xi-t)^{\nu-1} f(t) d t
$$

where $c$ and $\xi$ are the lower and upper limits of the integral. Accordingly, if $\nu=m-\mu>0$ and $\mu>0$, we have 


$$
D^{\mu} f(\xi)=D^{m}\left[D^{-\nu} f(\xi)\right], \text { where } \xi>0 .
$$

Consequently, we can define the fractional discrimination functions by combining the aggregation function $\alpha(\xi)$ with the fractional discrete derivatives.

Let $D^{\mu}$ denote the fractional discrete derivative operator (FDD). The fractional discrete derivative is performed by convolving the coefficients of FDD with the given image. The FDD coefficients for the order from 0.1 to 1.0 can be determined based on a Maclaurin series expansion of $(1-x)^{p}, p<1.0$. Higher order of FDD coefficients can be derived by repeated convolutions of the lower order of FDDs. The details of the related calculation are given in [7]. The fractional discrimination functions gradually phase out the contrasts and eventually result in a peak or zero-crossing map for the 1st and 2nd-order operation. As a result, subtle features in an image will be extracted at different resolutions in terms of various fractional-orders, which is very powerful in texture feature extraction.

\section{Experimental Results}

The fractional discrimination function detailed in this paper can be used for feature enhancement in different region boundaries for texture image segmentation. Figure 1 shows our comparative studies by detecting the coast line of the map of Australia which consists of two different but visually similar textures. Figure 1(a) is the original image with different textured regions. Figure 1(b)-1(c) are the segmented images by means of Prewitt edge detector and fractional discrimination function respectively. The advantage of our fractional functions to image enhancement is further demonstrated in Figure 2, where Figure 2(a) is the original spine image with noise, Figure 2(b) Figure 2(f) are the series of enhanced images corresponding to the fractional order of $0,0.2,0.4,0.5,0.8$ and 0.9 . The gradual transition between the original image and the first-order image enhancement makes it useful for medical diagnosis. Table 1 lists some statistical measurements made on the spine image. for the comparison of the performance at different fractional order.

Table 1: Statistical measurements at different fractional order

\begin{tabular}{|c|c|c|c|c|c|}
\hline order & Mean & SDV & Skeweness & Entropy & SNR \\
\hline 0.0 & 129.91 & 46.65 & -2.24 & 3.58 & 0.00 \\
\hline 0.2 & 131.75 & 47.43 & -2.21 & 3.79 & 22.78 \\
\hline 0.3 & 136.32 & 50.08 & -2.16 & 3.72 & 19.57 \\
\hline 0.4 & 138.79 & 50.55 & -2.18 & 3.90 & 19.28 \\
\hline 0.5 & 133.48 & 47.87 & -2.21 & 3.75 & 23.01 \\
\hline 0.6 & 134.07 & 48.01 & -2.22 & 3.50 & 23.91 \\
\hline 0.7 & 134.29 & 48.08 & -2.22 & 3.70 & 22.92 \\
\hline 0.8 & 137.47 & 48.14 & -2.22 & 3.81 & 22.91 \\
\hline 0.9 & 134.68 & 48.20 & -2.22 & 3.91 & 22.86 \\
\hline
\end{tabular}

\section{Conclusion}

In this paper we have outlined a method of extracting features from complex mix of texture patterns. The technique uses simple spatial fractional discrimination processes to enhance the embedded features. Using this method is it possible to extract features which cannot be normally be extracted using statistical and structural methods. The main advantage in using this approach, it is possible to enhance texture features by simple convolution operation.

\section{References}

[1] R.M. Haralick, "Statistical and structural approaches to texture", Proc. IEEE, Vol. 67, pp. 786-804, 1979.

[2] K.I. Laws, "Textured image segmentation", $P h . D$ thesis, University of Southern California, January, 1980.

[3] K.K. Benke, D.R. Skinner and C.J. Woodruff, "Convolution operators as a basis for objective correlates for texture perception", IEEE Trans. Syst., Man, Cybern., Vol. SMC-18, pp. 158-163, 1988.

[4] J. You and H.A. Cohen, "Classification and Segmentation of Rotated and Scaled Textured Images Using Texture 'Tuned' Masks", Pattern Recognition, Vol. 26, No. 2, Feb., pp. 245 - 258, 1993.

[5] L.S. Davis, "A survey of edge detection techniques", Comput. Graphics Image Process., Vol. 3, pp. 248-270, 1975.

[6] J.J. Koenderink and A.J. van Doorn, "Receptive Field Assembly Pattern Specificity", J. Visual Comm. Image Rep., Vol. 3, pp. 1-2, 1992.

[7] S. Hunganehally, On discrimination functions for image feature extraction, Ph.D thesis, Griffith University, 1997.

[8] K.S. Miller and B. Ross, "An introduction to fractional calculus and fractional differential equations", John Wiley and Sons, New York, 1994. 


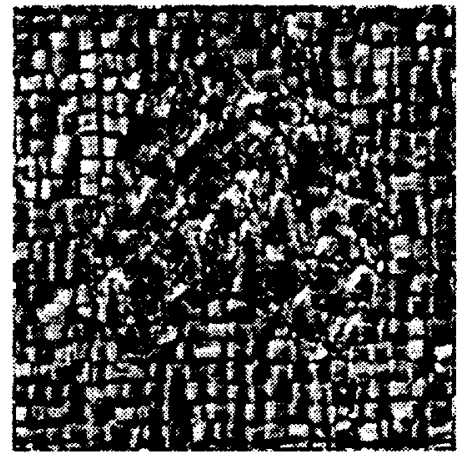

(a) texture image

(two-texture composite)

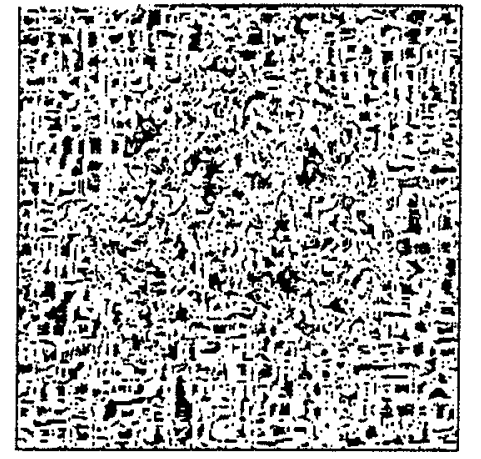

(b) edge image

(Prewitt operator)

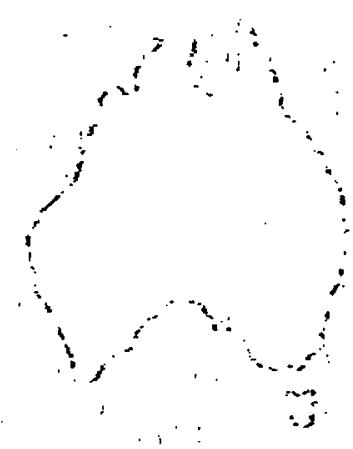

(c) enhanced image (fractional function)

Figure 1: Comparison of feature points for texture segmentation

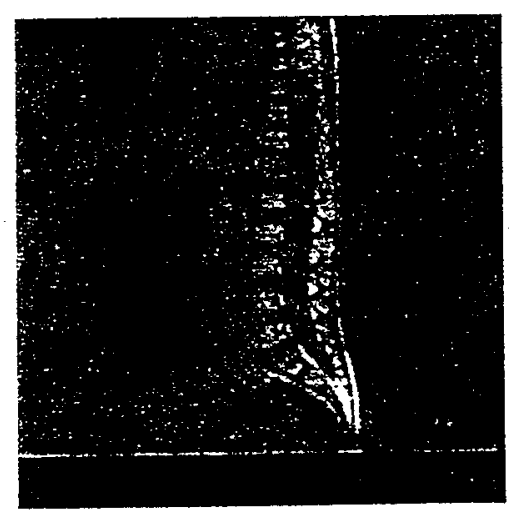

(a) original image

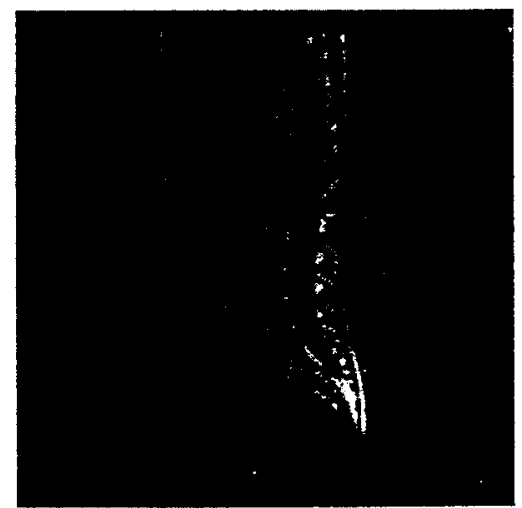

(d) fractional order $=0.5$

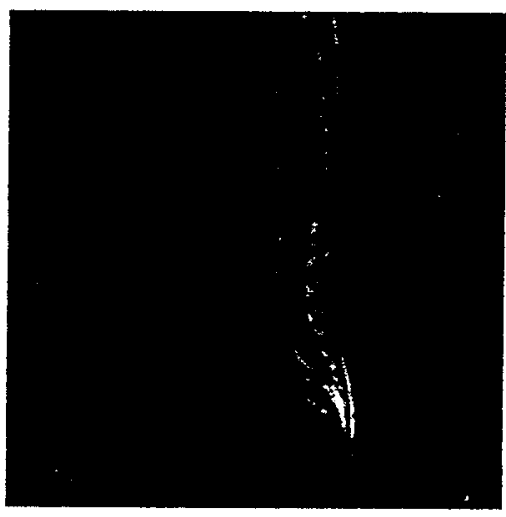

(b) fractional order $=0.2$

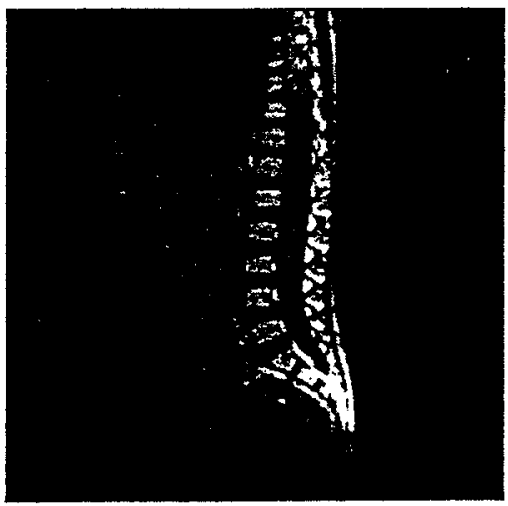

(e) fractional order $=0.8$

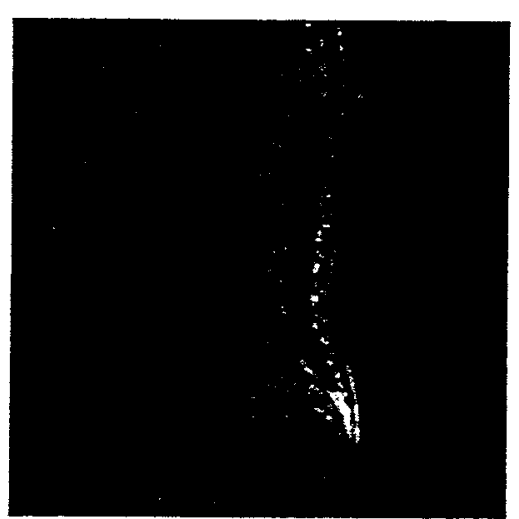

(c) fractional order $=0.4$

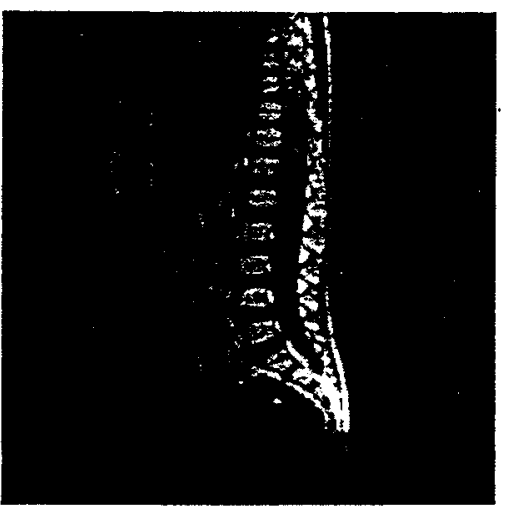

(f) fractional order $=0.9$

Figure 2: Fractional discrimination operation 